\title{
Short term improvement in exercise capacity and symptoms following exercise training in interstitial lung disease
}

\author{
A E Holland, ${ }^{1,2}$ C J Hill ${ }^{3,4}$ M Conron, ${ }^{5}$ P Munro, ${ }^{2}$ C F McDonald ${ }^{3,4}$
}

${ }^{1}$ School of Physiotherapy, La Trobe University, Melbourne, Australia; ${ }^{2}$ Bayside Health, Melbourne, Australia; ${ }^{3}$ Institute for Breathing and Sleep, Melbourne, Australia; ${ }^{4}$ Austin Health, Melbourne, Australia;

${ }^{5}$ St Vincent's Hospital,

Melbourne, Australia

Correspondence to:

Dr A Holland, Physiotherapy Department, Alfred Hospital, Commercial Rd, Melbourne,

Australia 3004; a.holland@ alfred.org.au

Received 30 July 2007 Accepted 21 December 2007 Published Online First

1 February 2008

\begin{abstract}
Background: Interstitial lung disease (ILD) is characterised by exertional dyspnoea, exercise limitation and reduced quality of life. The role of exercise training in this diverse patient group is unclear. The aims of this study were to establish the safety of exercise training in ILD; its effects on exercise capacity, dyspnoea and quality of life; and whether patients with idiopathic pulmonary fibrosis (IPF) had similar responses to those with other types of ILD.
\end{abstract}

Methods: 57 subjects with ILD (34 IPF) were randomised to receive 8 weeks of supervised exercise training or weekly telephone support. The 6 min walk distance (6MWD), incremental exercise test, modified Medical Research Council (MRC) dyspnoea score and Chronic Respiratory Disease Questionnaire (CRDO) were performed at baseline, following intervention and at 6 months.

Results: $80 \%$ of subjects completed the exercise programme and no adverse events were recorded. The GMWD increased following training (mean difference to control $35 \mathrm{~m}, 95 \% \mathrm{Cl} 6$ to $64 \mathrm{~m}$ ). A significant reduction in MRC score was observed (0.7 points, 95\% Cl 0.1 to 1.3) along with improvements in dyspnoea $(p=0.04)$ and fatigue $(p<0.01)$ on the CRDO. There was no change in peak oxygen uptake; however, exercise training reduced heart rate at maximum isoworkload $(p=0.01)$. There were no significant differences in response between those with and without IPF. After 6 months there were no differences between the training and control group for any outcome variable.

Conclusions: Exercise training improves exercise capacity and symptoms in patients with ILD, but these benefits are not sustained 6 months following intervention.

Trial registration number: NCT00168285

The interstitial lung diseases (ILDs) are a diverse group of chronic lung conditions characterised by dyspnoea on exertion and poor health related quality of life. ${ }^{1-3}$ Treatment options are often limited and patients suffer increasing exercise limitation as their disease progresses. Although exercise training effectively improves exercise capacity and health related quality of life in patients with chronic obstructive pulmonary disease (COPD), ${ }^{4}$ its role in patients with ILD is unclear. Small uncontrolled trials suggest positive effects of exercise training on endurance and quality of life, ${ }^{5}{ }^{6}$ but more robust evidence of benefit in ILD is lacking.

Current guidelines for exercise prescription in chronic lung disease are based on evidence from studies of patients with $\mathrm{COPD}^{7}$ where respiratory mechanics and peripheral muscle dysfunction are major limitations to exercise capacity. ${ }^{89}$ The applicability of such guidelines to patients with ILD is unclear as the physiological basis of exercise limitation differs substantially from COPD. Exercise performance in ILD is closely associated with circulatory impairment ${ }^{1011}$ and is characterised by exercise induced hypoxaemia that may be profound. ${ }^{12-14}$ Pulmonary arterial hypertension is also common ${ }^{15}$ and may worsen with exercise. ${ }^{16}$ In addition, ILD is a heterogeneous group of diseases. Patients with idiopathic pulmonary fibrosis (IPF) generally demonstrate greater abnormalities of exercise induced gas exchange than those with other forms of ILD..$^{18}$ Whether it is safe and efficacious to apply standard exercise training principles to all patients with ILD is unknown. Our aims in this study were therefore to evaluate: (1) the safety of a standard exercise training programme in ILD; (2) the effects of exercise training on exercise capacity, dyspnoea and quality of life; and (3) whether there is a difference in response to exercise training in IPF compared with other ILDs.

\section{METHODS}

A two site, randomised, single blinded controlled trial was conducted at the Alfred Hospital and Austin Health, Melbourne, Australia. Symptomatic patients aged over 18 years with documented ILD of any aetiology were invited to take part. The diagnosis of ILD was made according to established criteria. For IPF, the diagnostic criteria were consistent with those outlined in the International Consensus Statement. ${ }^{19}$

Patients were eligible to participate if they were ambulant and reported dyspnoea on exertion on stable medical therapy. Exclusion criteria were a history of syncope on exertion or any comorbidities which precluded exercise training (such as severe orthopaedic or neurological deficits or unstable cardiac disease). Patients were also excluded if they had participated in a pulmonary rehabilitation programme in the past 12 months. All subjects gave written informed consent and the study was approved by the human ethics committees at both trial sites. Random allocation to exercise and control groups was performed at a central location by an individual unrelated to the study who opened a sealed opaque envelope. Randomisation was performed separately for those subjects with IPF to ensure an even distribution across groups. 
Baseline measurements of spirometry, diffusing capacity and plethysmographic lung volumes were obtained. A transthoracic echocardiogram was performed to assess whether pulmonary hypertension was present. Spirometry and diffusing capacity measurements were repeated immediately after the intervention period and at 6 months.

The intervention group attended a twice weekly exercise training programme at the institution where they were recruited for a period of 8 weeks. Training was conducted according to the same exercise prescription principles as those used for COPD. T The exercise prescription consisted of $30 \mathrm{~min}$ of endurance exercise at each session, comprising both stationary cycling and walking training. Initial walking intensity corresponded to $80 \%$ of the walking speed achieved on the baseline 6 min walk test. Upper limb endurance training and functional strength training for the lower limbs were performed. Exercise was monitored and progressed by an experienced physiotherapist according to a standardised protocol. Supplemental oxygen was provided during training if necessary to achieve oxygen saturation $\geqslant 85 \%$. Once the subject was established on a supervised exercise regimen, an unsupervised home exercise programme was prescribed with the aim of achieving five exercise sessions per week in total. Subjects were considered to have completed the exercise programme if they attended 12 or more out of a possible 16 supervised exercise sessions. The control group did not receive a supervised exercise training programme but were contacted once a week by telephone during the 8 week period to provide support and general health advice.

The primary outcome, functional exercise capacity, was measured before and after the 8 week intervention period using the 6 min walk test. ${ }^{20}$ Use of oxygen during the test was

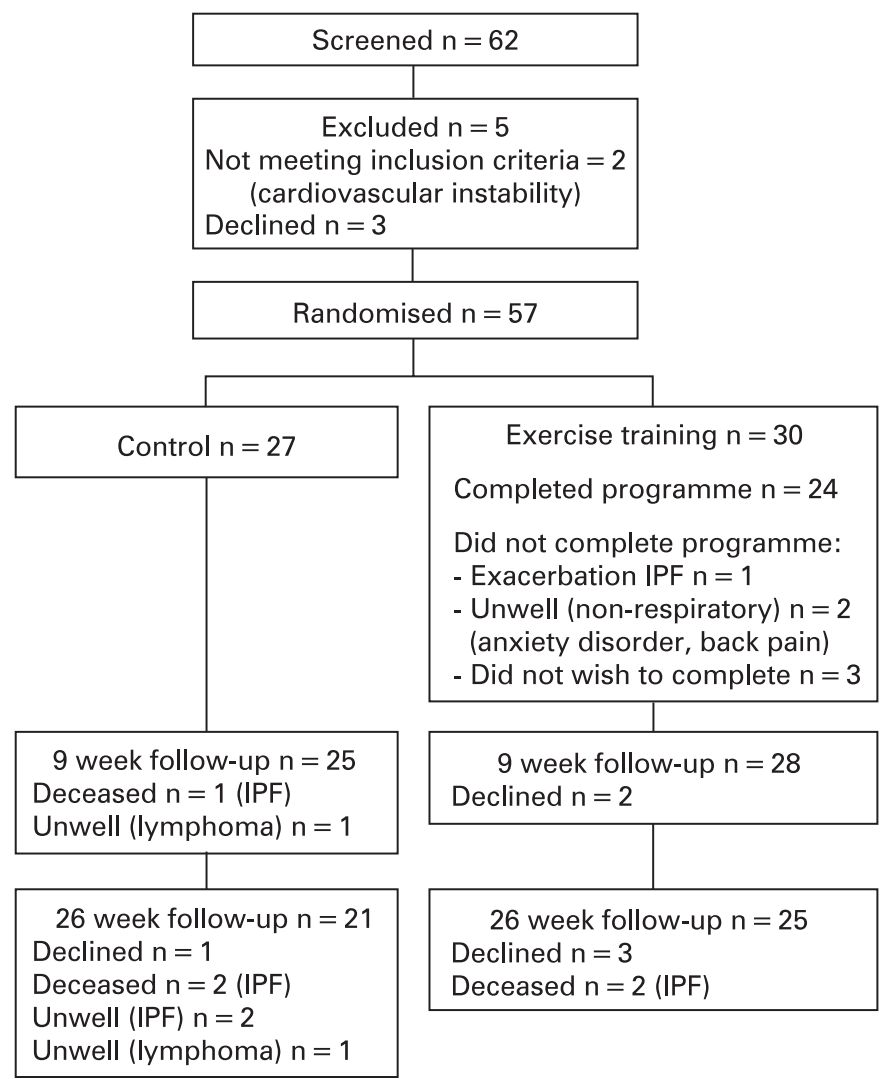

Figure 1 Participant flow. IPF, idiopathic pulmonary fibrosis. standardised and the test was stopped if desaturation to less than $80 \%$ occurred, in accordance with a previously described protocol for ILD. ${ }^{21}$ All follow-up walk tests were conducted using the same flow rate of supplemental oxygen that had been used at baseline. The distance walked in $6 \mathrm{~min}$ (6MWD), continuous walk velocity and lowest oxygen saturation were recorded.

Secondary outcomes of maximal exercise capacity, quality of life and dyspnoea were also measured at baseline and after completion of the 8 week intervention. An incremental exercise test was performed on a cycle ergometer in all subjects with baseline oxygen saturation greater than $85 \% .^{22}$ Peak oxygen uptake, minute ventilation, heart rate, workload and duration of the test were recorded. Heart rate and minute ventilation at isoworkload were calculated. Both generic and disease specific health related quality of life were assessed using the Medical Outcomes Study Short Form 36 (SF-36) and the Chronic Respiratory Disease Questionnaire (CRDQ). These questionnaires have previously been validated in ILD. ${ }^{3}$ Dyspnoea was assessed with the modified Medical Research Council (MRC) Scale. ${ }^{23}$ Following the intervention period, participants from both groups were asked to make a global rating of change in their walking ability as either "worse", "about the same" or "better". ${ }^{24}$ At 6 months, measurements of 6MWD, dyspnoea and quality of life were repeated. All measurements were obtained by an independent data collector who was blinded to treatment allocation.

The pre-specified sample size was 57 patients based on pilot data collected at our centres in 21 patients with ILD who underwent an exercise training programme. The mean observed change in 6MWD in this pilot study was $38 \mathrm{~m}$ (SD $43 \mathrm{~m}$ ). This sample size provided statistical power of $80 \%$ to detect changes in 6MWD $(\mathrm{n}=44)$ and all domains of the CRDQ $(\mathrm{n}=57)$. Data analysis was performed according to the intention-to-treat principle and attempts were made to obtain all available data points from all subjects. In the case of subjects being too unwell to perform exercise tests at follow-up, questionnaire data only were obtained. Subjects were considered to have completed the study if data for at least one outcome was available at 6 months. Missing data were replaced by the last-observationcarried-forward (LOCF) method. ${ }^{25}$ Subgroup analyses were specified a priori for subjects with and without IPF. To test the robustness of the LOCF analysis, a sensitivity analysis was performed using a conservative method where the observed means from the exercise and control groups were substituted for missing data in the opposite arm. ${ }^{26}$ The sensitivity analysis confirmed the results of the primary endpoint and hence the LOCF analyses have been reported.

Statistical analysis was performed using a two way analysis of variance to account for group and time interaction (SPSS V.14.0; SPSS Inc). Post-hoc contrasts were specified for differences between groups at 9 weeks and 26 weeks. The significance level was set at $p<0.05$ and differences between groups expressed as means (SD) or $95 \%$ confidence intervals (95\% CI).

\section{RESULTS}

Between February 2005 and November 2006, 62 patients were screened and 57 patients were randomly assigned to treatment (fig 1). Thirty-four subjects $(60 \%)$ had a diagnosis of IPF. Of these, 12 subjects had a confirmed pattern of usual interstitial pneumonia on open or thorascopic lung biopsy and the remainder had typical findings of usual interstitial pneumonia on high resolution computed tomography. Four subjects had 
Table 1 Baseline characteristics of the study population

\begin{tabular}{lcc}
\hline & Control & Exercise \\
\hline Age (y) & $67(13)$ & $70(8)$ \\
FVC (\%pred) & $75(25)$ & $77(24)$ \\
Tlco (\%pred) & $49(18)$ & $50(19)$ \\
TLC (\%pred) $(\mathrm{n}=49)$ & $76(22)$ & $76(17)$ \\
RVSP (mm Hg) $(\mathrm{n}=43)$ & $38(13)$ & $37(15)$ \\
6MWD (m) & $395(139)$ & $375(116)$ \\
Vo $0_{2}$ peak (\%pred) $(\mathrm{n}=52)$ & $58(15)$ & $55(19)$ \\
MRC dyspnoea & $3(1)$ & $3(1)$
\end{tabular}

Data are mean (SD).

Data from all 57 subjects except where indicated.

6MWD, 6 min walk distance; \%pred, per cent predicted; FVC, forced vital capacity; MRC, modified Medical Research Council dyspnoea scale; RVSP, right ventricular systolic pressure; TLC, total lung capacity; Tlco, diffusing capacity for carbon monoxide; $\mathrm{V}_{2}$ peak, peak oxygen uptake.

idiopathic interstitial pneumonias other than IPF (non-specific interstitial pneumonia, cryptogenic organising pneumonia). Of the remaining subjects, 14 had diffuse parenchymal lung disease of known cause (collagen vascular disease, drug or dust related), four subjects had granulomatous lung disease (sarcoidosis, Wegener's granulomatosis) and one subject Langerhans cell histiocytosis.

Baseline characteristics of the 57 subjects are shown in table 1. There were no differences between the groups at baseline in demographic variables or in the proportion of subjects with IPF. Exercise capacity did not differ between the groups at baseline either in terms of $6 \mathrm{MWD}$ or peak oxygen uptake $\left(\mathrm{VO}_{2}\right.$ peak). The mean 6MWD at baseline in subjects with IPF was 354 (SD 125) $\mathrm{m}$ compared with a mean of 431 (117) $\mathrm{m}$ in those without $\operatorname{IPF}(p=0.02)$.

Forty-six subjects completed the study (fig 1). There was a significant reduction in FVC over the 6 month study period (mean reduction $220 \mathrm{ml}, 95 \% \mathrm{Cl} 60$ to $380 \mathrm{ml} ; \mathrm{p}=0.047$ ). There was no difference between groups in the magnitude of reduction in FVC. There was no change in the diffusing capacity for carbon monoxide (Tlco) over the study period. There were two deaths in each group, with one death in the control group prior to 9 weeks and the remaining three deaths occurring during the follow-up period. All deceased subjects had a diagnosis of IPF.

Twenty-four subjects completed the exercise programme $(80 \%)$. Reasons for non-completion are shown in fig 1 . No adverse events were recorded during exercise training.

The effects of exercise training on the primary outcome of $6 \mathrm{MWD}$ are shown in fig 2. Data were imputed for two patients in each group at 9 weeks; at 6 months, data were imputed for eight subjects in the control group and seven subjects in the exercise group. There was a significant interaction between group and time for walk distance $(p=0.01)$. The $6 \mathrm{MWD}$ improved in the exercise group after the training programme, with a mean increase compared with controls of $35 \mathrm{~m}$ (table 2). However, this effect was not sustained at 6 months. A similar interaction was seen between group and time for walk velocity $(p=0.003)$, with a mean increase of $0.33 \mathrm{~km} / \mathrm{h}$ in the exercise group following training (table 2) which was not sustained at 6 months. There were no differences between the groups for baseline oxygen saturation or lowest recorded oxygen saturation during the test at any time point.

Cardiopulmonary exercise testing was performed in 52 subjects at baseline, with data imputed at 9 weeks for five subjects in the control group and three subjects in the exercise group. Changes in indices of cardiopulmonary performance are

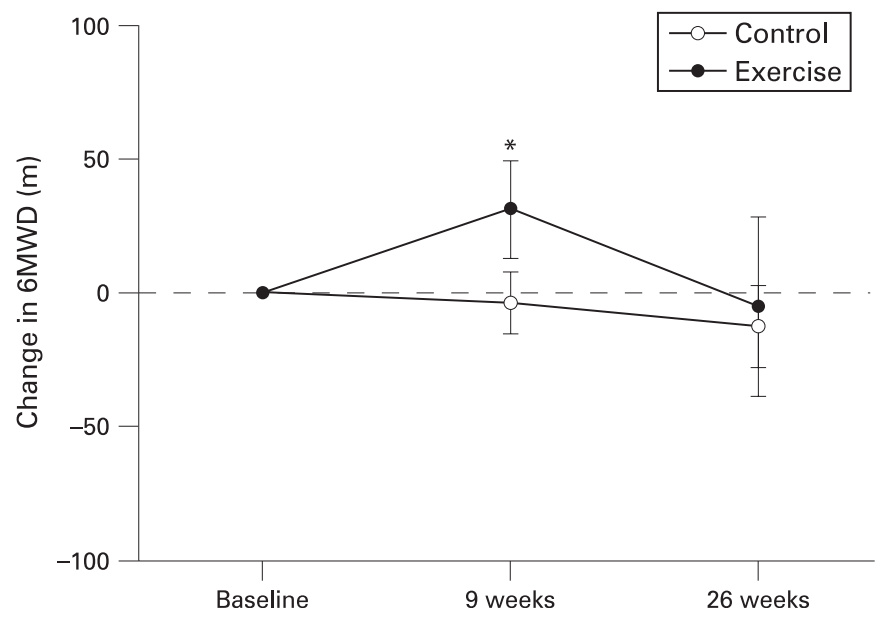

Figure 2 Change in 6 minute walk distance (6MWD) test. Data are means and $95 \% \mathrm{Cl}$. ${ }^{*} \mathrm{p}<0.05$, exercise vs control group.

shown in table 2. There were no significant differences between groups in $\mathrm{VO}_{2}$ peak, maximum heart rate or minute ventilation following training. The exercise group showed a reduction in heart rate at maximum isoworkload following training $(p=0.01)$.

Data for dyspnoea and quality of life outcomes were imputed for two subjects in each group at 9 weeks and five subjects in each group at 6 months. There was a significant interaction between group and time for modified MRC dyspnoea score $(p=0.04)$ (fig 3$)$. There was a reduction in modified MRC score following exercise training with a mean difference between groups of 0.7 points (table 2). However, this reduction did not persist at 6 months. The CRDQ also demonstrated improvements following exercise training in dyspnoea $(p=0.04)$ and fatigue $(p=0.009)$ (table 2$)$. These effects were no longer statistically significant at 6 months (fig 4). There were no statistically significant changes in emotional function or mastery over time. Of the eight domains of the SF-36, only vitality showed gains immediately following exercise training

Table 2 Differences between the exercise and control groups following intervention

\begin{tabular}{|c|c|c|}
\hline & $\begin{array}{l}\text { Mean difference } \\
(95 \% \mathrm{CI})\end{array}$ & p Value \\
\hline 6MWD (m) & $35(6-64)$ & 0.01 \\
\hline Walk velocity $(\mathrm{km} / \mathrm{h})$ & $0.33(0.01-0.65)$ & 0.003 \\
\hline $\mathrm{V}_{0_{2}}$ peak $(\mathrm{ml} \times \mathrm{kg} / \mathrm{min})$ & $0.70(-0.30-1.68)$ & 0.16 \\
\hline HRmax (bpm) & $1.8(-2.8-6.5)$ & 0.43 \\
\hline V̇Emax (I/min) & $4.7(-0.1-9.5)$ & 0.06 \\
\hline Wmax (W) & $3.5(-0.44-7.39)$ & 0.08 \\
\hline Test duration (min) & $0.91(-0.04-1.86)$ & 0.06 \\
\hline HR isowork (bpm) & $-6.6(-11.7--1.52)$ & 0.01 \\
\hline$\dot{V}_{E}$ isowork (I/min) & $-1.4(-5.94-3.1)$ & 0.53 \\
\hline MRC dyspnoea score & $0.7(0.1-1.3)$ & 0.04 \\
\hline CRDO Dyspnoea & $3.1(0.1-6.1)$ & 0.04 \\
\hline CRDO Fatigue & $2.9(0.8-5.0)$ & 0.009 \\
\hline CRDO Mastery & $1.8(-0.2-3.7)$ & 0.07 \\
\hline CRDO Emotional function & $3.7(-0.2-8.2)$ & 0.10 \\
\hline
\end{tabular}

Data are exercise group - control group.

6MWD, six minute walk distance; CRDQ, Chronic Respiratory Disease Questionnaire; $\mathrm{HR}$ isowork, heart rate at maximum isoworkload; HRmax, maximum heart rate; MRC modified Medical Research Council dyspnoea score; $\dot{V}_{E}$ isowork, ventilation at maximum isoworkload; $\dot{V}$ Emax, maximum minute ventilation; $\mathrm{Vo}_{2}$ peak, peak oxygen uptake; Wmax, maximum workload. 


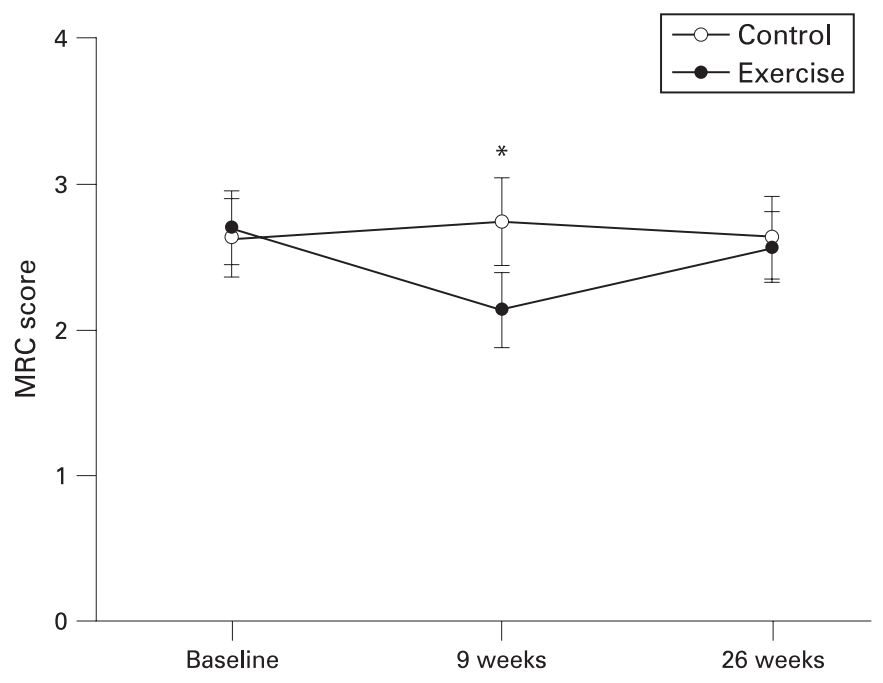

Figure 3 Modified Medical Research Council (MRC) dyspnoea scale. Data are means $(95 \% \mathrm{Cl}) .{ }^{*} \mathrm{p}<0.05$ exercise vs control group.

( 3.58 points, 0.44 to $5.72 ; p=0.005$ ) but this difference was not evident at 6 months.

Global rating of change scores indicated that $68 \%$ of participants in the exercise group perceived that their walking ability had improved during the intervention period compared with $20 \%$ of participants in the control group. The proportions of improved participants were similar in the subgroup of patients with IPF $(73 \%$ in the exercise group, $20 \%$ in the control group).

Subgroup analyses showed that the mean improvement in 6MWD for subjects with IPF was 25.1 (54.2) m compared with 43.5 (34.2) $\mathrm{m}$ in those without IPF; however, there was wide variation in response and this did not reach statistical significance $(p=0.34)$. The decline in $6 \mathrm{MWD}$ from 9 weeks to 26 weeks in those with IPF was -44.2 (75.4) m compared with -20.8 (35.6) $\mathrm{m}$ in those without IPF. This difference was not statistically significant $(p=0.65)$. No significant differences between the response of those with and without IPF were evident for any outcome measure.

\section{DISCUSSION}

This study is the first randomised controlled trial of exercise training in ILD. We have shown that exercise training is safe and feasible in this patient group, with programme completion rates similar to those reported in COPD.? Short term gains in functional exercise capacity and improved symptoms were demonstrated following exercise training using a standard pulmonary rehabilitation training protocol. However, the 6 month follow-up indicates that these benefits were not sustained over time.

Our data showed a mean improvement in 6MWD following exercise training of $35 \mathrm{~m}$. This is a smaller gain than that reported in previous trials of pulmonary rehabilitation performed in patients with COPD, where increases of approximately 50 m were common. ${ }^{4}$ We used the same exercise training principles that are applied in $\mathrm{COPD}^{7}$ and we have previously shown that our programme achieves gains in 6MWD which exceed the minimal clinically important difference in patients with COPD. ${ }^{27}$ It therefore seems likely that the magnitude of response to pulmonary rehabilitation is smaller in patients with ILD. Given the progressive nature of many ILDs and the evidence of disease progression in our subjects, a smaller degree of change following exercise training is not unexpected.

The minimal clinically important difference for the 6MWD in ILD has not been defined and the clinical significance of our finding is therefore unclear. Our results could be compared with
Figure 4 Health related quality of lifeChronic Respiratory Disease Questionnaire. Data are means $(95 \% \mathrm{CI})$. ${ }^{*} p<0.05$ exercise vs control group.
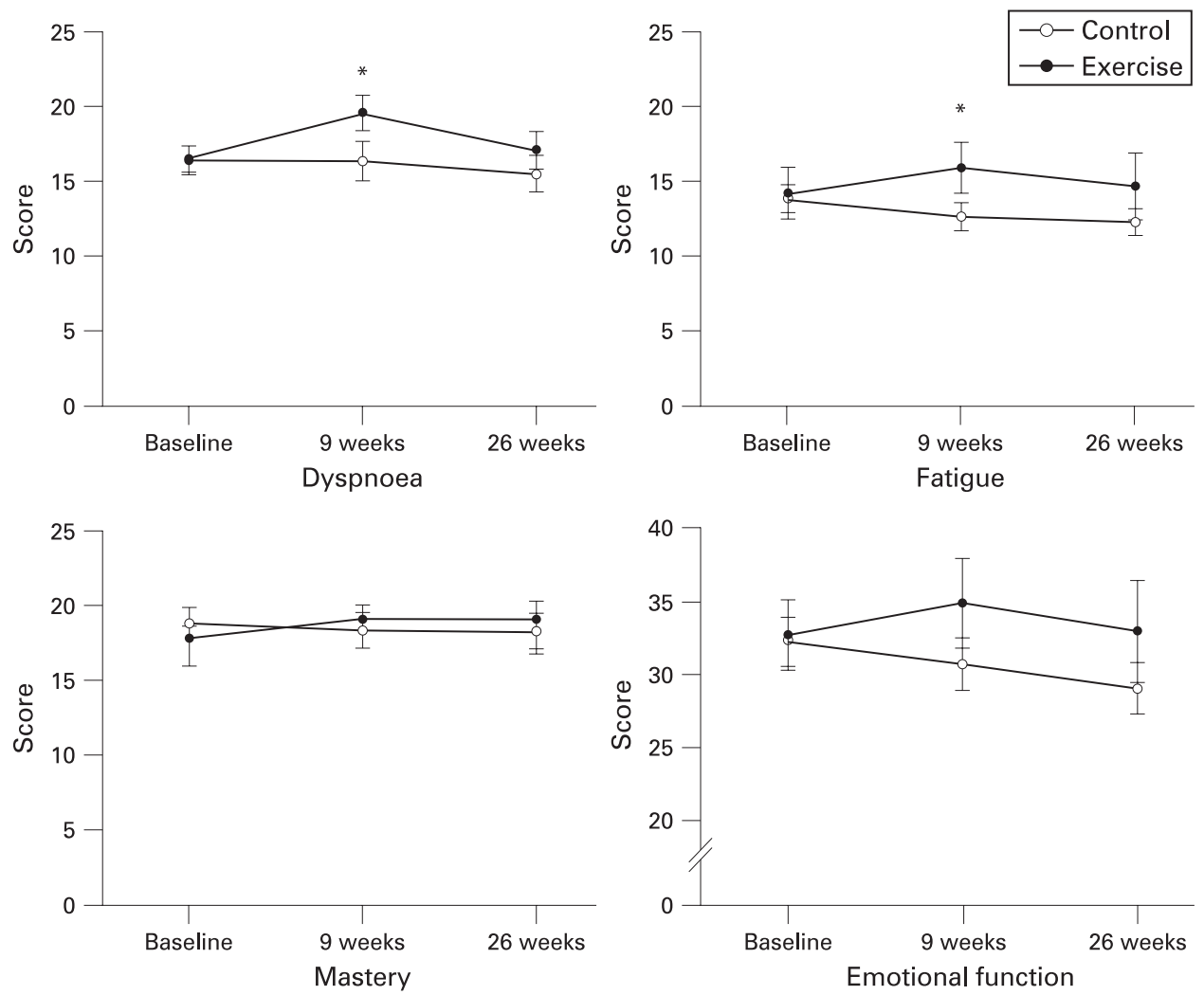
a recent uncontrolled report of a mean improvement in 6MWD of $49 \mathrm{~m}$ following sildenafil therapy in IPF. ${ }^{28}$ The observed gains in exercise capacity in our trial were associated with improvements in dyspnoea and fatigue on the CRDO as well as improved global rating of change scores in the majority of participants. This suggests that clinically relevant improvement in symptoms did occur in most participants following exercise training.

The improvements in symptoms and walking distance reported here did not occur as a result of improved maximum exercise capacity. No differences in $\mathrm{Vo}_{2}$ peak or any other maximum exercise variables were observed following training. However, the significant reduction in heart rate at maximum isoworkload indicates that a cardiovascular adaptation to training was achieved. This suggests that while maximum exercise capacity may be constrained by the pathophysiology of ILD, submaximal exercise capacity is amenable to training. It is also likely that peripheral muscle adaptation contributed significantly to improved functional capacity. Peripheral muscle weakness is predictive of exercise intolerance in ILD $^{29} 30$ and previous studies in COPD have indicated that much of the improvement in exercise capacity following pulmonary rehabilitation is due to peripheral muscle adaptation. ${ }^{31}{ }^{32}$ A limitation of this study is that peripheral muscle strength was not measured and future studies of exercise training in ILD should include this variable.

The lack of a benefit of exercise training after 6 months indicates that the observed improvements in exercise capacity and symptoms may only be of short duration. Previous studies have shown that the benefits of pulmonary rehabilitation decline over 6-12 months but differences to the control group are still evident after 1 year. ${ }^{33}$ Our study was performed in subjects with progressive lung disease, which may explain the faster decline in benefit. The pulmonary rehabilitation literature suggests that health related quality of life is better preserved than is exercise capacity. ${ }^{34}{ }^{35}$ Our data reflect this pattern; but differences at 6 months were not statistically significant. The number of dropouts at 6 months may have affected these results and is a common difficulty in trials which include participants with IPF. ${ }^{36}$ Participants in our trial were encouraged to continue with home exercise but did not undertake a formal maintenance exercise programme. It is possible that the benefits of exercise training in ILD may have been longer lasting if a maintenance intervention had been employed; however, at present, the role of maintenance programmes following initial structured programmes remains unclear. ${ }^{37}$

It is a limitation of this study that the effects of disease aetiology and severity on response to exercise training could not be fully characterised. Interstitial lung disease is a heterogeneous group of conditions with variable disease progression. Although our subjects with IPF appeared to have smaller gains in exercise capacity and health related quality of life than those with other forms of ILD, our study was not powered to adequately assess this outcome. Our sample included subjects with a wide range of disease severity, with diffusing capacity for carbon monoxide ranging from $19 \%$ to $75 \%$ predicted and right ventricular systolic pressure ranging from 16 to $80 \mathrm{~mm} \mathrm{Hg}$. The small sample size did not permit analysis of the effects of disease severity on outcomes of exercise training. Further research with larger samples is needed to address the effects of aetiology on response to training and to establish whether there is an optimal time in the disease course at which exercise training should be offered. This is particularly important for patients with IPF, where the prognosis is poor and the burden of care must be carefully considered.

Treatment options for ILD are limited. Available drug therapy has significant toxic side effects and may not be suitable for many patients. There is no evidence that current drug therapies for IPF can improve quality of life and symptoms. ${ }^{38}$ In this setting, the modest improvements and absence of side effects associated with exercise training may be of importance to some patients. The short term beneficial effects of exercise training on dyspnoea and 6MWD, variables which are important to prognosis, ${ }^{12}$ suggest that further investigation of exercise training is warranted in larger trials.

In conclusion, exercise training in interstitial lung disease is safe and results in short term improvement in functional exercise capacity, dyspnoea and quality of life. These benefits are no longer evident at 6 months. Exercise training may be considered in patients with interstitial lung disease of any aetiology if dyspnoea is associated with diminished functional capacity.

Funding: This study was funded by the Victorian Tuberculosis and Lung Association. Competing interests: None.

Ethics approval: The study was approved by the human ethics committees at the Alfred Hospital and Austin Health, Melbourne, Australia.

\section{REFERENCES}

1. Collard HR, King TE Jr, Bartelson BB, et al. Changes in clinical and physiologic variables predict survival in idiopathic pulmonary fibrosis. Am J Respir Crit Care Med 2003;168:538-42.

2. Flaherty KR, Andrei AC, Murray $S$, et al. Idiopathic pulmonary fibrosis: prognostic value of changes in physiology and six-minute-walk test. Am J Respir Crit Care Med 2006;174:803-9.

3. Chang JA, Curtis JR, Patrick DL, et al. Assessment of health-related quality of life in patients with interstitial lung disease. Chest 1999;116:1175-82.

4. Lacasse $\mathbf{Y}$, Goldstein R, Lasserson TJ, et al. Pulmonary rehabilitation for chronic obstructive pulmonary disease. Cochrane Database Syst Rev 2006:CD003793.

5. Naji NA, Connor MC, Donnelly SC, et al. Effectiveness of pulmonary rehabilitation in restrictive lung disease. J Cardiopulm Rehabil 2006;26:237-43.

6. Foster $\mathbf{S}$, Thomas HM 3rd. Pulmonary rehabilitation in lung disease other than chronic obstructive pulmonary disease. Am Rev Respir Dis 1990;141:601-4.

7. Nici L, Donner C, Wouters E, et al. American Thoracic Society/European Respiratory Society statement on pulmonary rehabilitation. Am J Respir Crit Care Med 2006;173:1390-413.

8. O'Donnell DE, Revill SM, Webb KA. Dynamic hyperinflation and exercise intolerance in chronic obstructive pulmonary disease. Am J Respir Crit Care Med 2001;164:7707.

9. Gosselink R, Troosters T, Decramer M. Peripheral muscle weakness contributes to exercise limitation in COPD. Am J Respir Crit Care Med 1996;153:976-80.

10. Hansen JE, Wasserman K. Pathophysiology of activity limitation in patients with interstitial lung disease. Chest 1996;109:1566-76.

11. Harris-Eze A0, Sridhar G, Clemens RE, et al. Role of hypoxemia and pulmonary mechanics in exercise limitation in interstitial lung disease. Am J Respir Crit Care Med 1996;154:994-1001.

12. Chetta A, Aiello M, Foresi A, et al. Relationship between outcome measures of sixminute walk test and baseline lung function in patients with interstitial lung disease. Sarcoidosis Vasc Diffuse Lung Dis 2001;18:170-5.

13. Lama VN, Flaherty KR, Toews GB, et al. Prognostic value of desaturation during a 6minute walk test in idiopathic interstitial pneumonia. Am J Respir Crit Care Med 2003;168:1084-90.

14. Risk C, Epler GR, Gaensler EA. Exercise alveolar-arterial oxygen pressure difference in interstitial lung disease. Chest 1984;85:69-74.

15. Lettieri CJ, Nathan SD, Barnett SD, et al. Prevalence and outcomes of pulmonary arterial hypertension in advanced idiopathic pulmonary fibrosis. Chest 2006;129:74652.

16. Bush A, Busst CM. Cardiovascular function at rest and on exercise in patients with cryptogenic fibrosing alveolitis. Thorax 1988;43:276-83.

17. Agusti AG, Roca J, Rodriguez-Roisin R, et al. Different patterns of gas exchange response to exercise in asbestosis and idiopathic pulmonary fibrosis. Eur Respir J 1988;1:510-16.

18. Wells AU, Hansell DM, Rubens MB, et al. Functional impairment in lone cryptogenic fibrosing alveolitis and fibrosing alveolitis associated with systemic sclerosis: a comparison. Am J Respir Crit Care Med 1997;155:1657-64.

19. Idiopathic pulmonary fibrosis. Diagnosis and treatment. international consensus statement. Am J Respir Crit Care Med 2000;161:646-64. 
20. ATS statement: guidelines for the six-minute walk test. Am J Respir Crit Care Med 2002;166:111-17.

21. Hallstrand TS, Boitano LJ, Johnson WC, et al. The timed walk test as a measure of severity and survival in idiopathic pulmonary fibrosis. Eur Respir J 2005:25:96-103.

22. ATS/ACCP Statement on cardiopulmonary exercise testing. Am J Respir Crit Care Med 2003;167:211-77.

23. Mahler D, Wells C. Evaluation of clinical methods for rating dyspnea. Chest 1988;93:580-6.

24. Jaeschke R, Singer J, Guyatt GH. Measurement of health status. Ascertaining the minimal clinically important difference. Control Clin Trials 1989;10:407-15.

25. Lachin JM. Statistical considerations in the intent-to-treat principle. Control Clin Trials 2000;21:167-89.

26. Miller ME, Morgan TM, Espeland MA, et al. Group comparisons involving missing data in clinical trials: a comparison of estimates and power (size) for some simple approaches. Stat Med 2001:20:2383-97.

27. Holland AE, Hill CJ, Nehez E, et al. Does unsupported upper limb exercise training improve symptoms and quality of life for patients with chronic obstructive pulmonary disease? J Cardiopulm Rehabil 2004;24:422-7.

28. Collard HR, Anstrom KJ, Schwarz MI, et al. Sildenafil improves walk distance in idiopathic pulmonary fibrosis. Chest 2007:131:897-9.

29. Spruit MA, Thomeer MJ, Gosselink R, et al. Skeletal muscle weakness in patients with sarcoidosis and its relationship with exercise intolerance and reduced health status. Thorax 2005:60:32-8.
30. Nishiyama $\mathbf{0}$, Taniguchi $\mathrm{H}$, Kondoh $\mathrm{Y}$, et al. Quadriceps weakness is related to exercise capacity in idiopathic pulmonary fibrosis. Chest 2005;127:2028-33.

31. Sala E, Roca J, Marrades RM, et al. Effects of endurance training on skeletal muscle bioenergetics in chronic obstructive pulmonary disease. Am J Respir Crit Care Med 1999;159:1726-34.

32. Whittom F, Jobin J, Simard PM, et al. Histochemical and morphological characteristics of the vastus lateralis muscle in patients with chronic obstructive pulmonary disease. Med Sci Sports Exerc 1998;30:1467-74.

33. Ries AL, Kaplan RM, Limberg TM, et al. Effects of pulmonary rehabilitation on physiologic and psychosocial outcomes in patients with chronic obstructive pulmonary disease. Ann Intern Med 1995;122:823-32.

34. Guell $\mathbf{R}$, Casan $P$, Belda J, et al. Long-term effects of outpatient rehabilitation of COPD: A randomized trial. Chest 2000;117:976-83.

35. Foglio K, Bianchi L, Bruletti G, et al. Long-term effectiveness of pulmonary rehabilitation in patients with chronic airway obstruction. Eur Respir J 1999;13: 125-32.

36. Johnson WC, Raghu G. Clinical trials in idiopathic pulmonary fibrosis: a word of caution concerning choice of outcome measures. Eur Respir J 2005;26: 755-8

37. Ries AL, Bauldoff GS, Carlin BW, et al. Pulmonary Rehabilitation: Joint ACCP/ AACVPR Evidence-Based Clinical Practice Guidelines. Chest 2007:131:4S-42.

38. Swigris JJ, Kuschner WG, Kelsey JL, et al. Idiopathic pulmonary fibrosis: challenges and opportunities for the clinician and investigator. Chest 2005;127:275-83.

\section{Pulmonary puzzle}

\section{ANSWER}

From the question on page 492.

Macroscopic findings favoured a diagnosis of mesothelioma. Microscopy revealed instead that the pleural thickening comprised fibrosis within which abundant CD68-positive histiocytes and Touton-type giant cells were present, these staining with S-100 but not CD1a. There was also microscopic involvement of the lung along the distribution of the lymphatic vessels and areas of yellow firm thickening in the epicardial fat adjacent to the coronary arteries demonstrating identical pathology. A diagnosis of Erdheim-Chester disease (ECD) was made.

ECD is a non-Langerhans histiocytic disorder primarily affecting bone. Over $50 \%$ of cases show involvement of other tissues including the retroperitoneum, retro-orbital tissues, heart, pituitaryhypothalamic axis and lung. ${ }^{1}$ Diagnosis is usually based on long bone radiographic changes. ${ }^{1-4}$ Bone scanning reveals symmetrical and abnormally increased distal lower limb long bone uptake. Pulmonary CT findings include distinctive interlobular and visceral pleural thickening associated with patchy reticular and centrilobular opacities and areas of ground-glass attenuation. ${ }^{4}$ Histopathologically, lung involvement in ECD is characterised by the accumulation of foamy or clear histiocytes with variable amounts of fibrosis and lymphoplasmacytic inflammatory infiltrate arranged in a lymphangitic pattern. ${ }^{1}$ Immunohistochemistry shows consistent staining for CD68 and negative staining for CD1a. Ultrastructural studies reveal an absence of Birbeck granules. ${ }^{2}$

Pulmonary ECD is typically diagnosed by a constellation of radiographic evidence and other key characteristic visceral signs with a virtually pathognomic bone profile. There is only one other case report of ECD mimicking mesothelioma. ${ }^{5}$ Diagnosis could only be made from microscopy at necropsy, highlighting the importance of tissue diagnosis rather than relying on macroscopic features alone.

Thorax 2008;63:554. doi:10.1136/thx.2007.91074a

\section{REFERENCES}

1. Egan AJ, Boardman LA, Tazelaar HD, et al. Erdheim-Chester disease: clinical, radiologic and histopathologic findings in five patients with interstitial lung disease. Am J Surg Pathol 1999;23:17-26.

2. Allen TC, Chevez-Barrios P, Shetlar DJ, et al. Pulmonary and ophthalmic involvement with Erdheim-Chester disease: a case report and review of the literature. Arch Pathol Lab Med 2004;128:1428-31.

3. Dion $\mathbf{E}$, Graef $\mathrm{C}$, Miquel A, et al. Bone involvement in Erdheim-Chester disease: imaging findings including periostitis and partial epiphyseal involvement. Radiology 2006:238:632-9.

4. Rush WL, Andriko JA, Galateau-Salle F, et al. Pulmonary pathology of Erdheim-Chester disease. Mod Pathol 2000;13:747-54

5. Saboerali MD, Koolen MG, Noorduyn LA, et al. Pleural thickening in a construction worker: it is not always mesothelioma. Neth J Med 2006:64:88-90. 